\title{
Microbiological evaluation of the antibacterial Vicryl suture in the mandibular third molar surgery
}

\author{
Milad Etemadi Sh${ }^{1}$, Sameen Rahgozar ${ }^{2}$, Golnaz Tajmiri ${ }^{3}$, \\ Javad Alizargar ${ }^{4}$, Shu-Fang Vivienne $\mathrm{Wu}^{5}$
}

1 Department of Oral and Maxillofacial Surgery, Dental Implants Research Center, Dental Research Institute, School of Dentistry, Isfahan University of Medical Sciences, Isfahan, Iran; Etemadi@dnt.mui.ac.ir

2 Department of oral and maxillofacial surgery, Faculty of Dentistry, Isfahan University of Medical Sciences, Isfahan,

Iran; Sameenrah@gmail.com

3 Students' Research Committee, School of Dentistry, Isfahan University of Medical Sciences, Isfahan, Iran; gtajmiri.gt@gmail.com

4 Research Center for Healthcare Industry Innovation, National Taipei University of Nursing and Health Sciences, Taipei City 112, Taiwan; jaz.tmu@gmail.com

5 College of Nursing, School of Nursing, National Taipei University of Nursing and Health Sciences, Taipei City 112, Taiwan; shufang@ntunhs.edu.tw

\begin{abstract}
:
Purpose: Selection and application of suture materials, has gained more importance especially with the increasing number of patients seeking oral surgeries. Since lying in a bacterial-filled environment, sutures make the tissue prone to infection. Suture material plays an important role in the reduction of the risk of infection. This study aimed to assess the success rate of an antibacterial suture named Vicryl Plus in preventing bacterial growth in the surgical site of the mandibular third molar. Materials and Methods: 27 patients were included in this double-blinded randomized clinical trial study. Surgical Extraction of the mandibular wisdom tooth was done and the incision was managed by randomly using Vicryl Plus and Vicryl sutures. After 7 days, sutures were removed and assessed microbiologically. Predominant species of Streptococcus mutans and Lactobacillus were assessed as well as the total number of colonies on each suture. Results: There was a significant difference between two suture materials in colony number-length ratio of lactobacillus $(\mathrm{p}$-value $=0.031)$ and total
\end{abstract}


bacterial colonies ( $p$-value $=0.016)$; but not for $S$. mutans species ( $p$-value $=0.201)$.

Conclusion: Antibacterial Vicryl suture can be a useful tool for the reduction in the rate of surgical site infection in high-risk cases and situations.

\section{Keywords: Suture material; Third molar surgery; Surgical wound infection; Bacterial adhesion}

\section{Introduction:}

The mandibular third molar is the most common tooth extracted by surgical procedures[1] which causes an interruption in tissue integrity, leading to wound formation[2]. For maximal wound repair and decreasing morbidity, it is necessary for the surgeons to have enough information about major factors that affect the wound repair[3]. One of these major factors is the selection of suture material[4] which may be the cause of wound infection by improper utilization[5-7]. It also can be the route of bacterial transmission to the wound site due to the bacterial adhesion[8]. This bacterial adhesion in part depends on the bacterial species, characteristics, and chemical structure of the suture material[ 7,8$]$.

Silk is the most commonly used suture material in oral surgery. Silk is cheaper than other materials and also easy to manipulate[9], but on the other hand, silk is comparatively prone to biofilm attachment and bacterial colonization. For these reasons, in the last two decades, oral surgeons have been seeking a better replacement for the silk sutures[10]. In recent years, antibacterial-coated sutures were manufactured and introduced to the market and are used without adequate clinical studies and scientific evidence.

Triclosan (5-chloro-2-(2,4dichlorophenoxy) phenol: TCS) is a halogenated phenol and a broad-spectrum antimicrobial agent which is used all over North America, Europe, and Asia as an agent in disinfectant materials, soap, toothpaste, mouth rinse, cloth, deodorant, shampoo and as an additive to plastic materials. Triclosan is an effective agent against many species of bacteria and special kinds of fungi. It penetrates the cell wall and by targeting multiple 
cytoplasmic and membrane receptors; makes changes in bacterial RNA synthesis and macromolecule production, which interferes with bacterial growth and causes cellular death[11].

Cruz et al. [10] assessed the effect of manipulation of sutures coated with antiseptic ointment (iodoform + calendula) in the reduction of bacterial colonization in a clinical trial study on 40 volunteer patients. The patients had randomly divided into two case and intervention groups. Two millimeters $(\mathrm{mm})$ of the suture were removed on the first and $15^{\text {th }}$ day after the surgery and they evaluated the colony units per milliliters $(\mathrm{CFU} / \mathrm{ml})$ in each group. There was a significant decrease in bacterial growth in the case group $(\mathrm{p}=0.002)$. So, they concluded that the antiseptic ointment is effective in bacterial growth decline on silk sutures.

In 2011, Galal et al.[12] evaluated the effect of coating the suture materials with triclosan, as an antibacterial agent, on the incidence of infection in the surgical sites. In this prospective randomized double-blinded study, the total number of 450 patients had divided into two groups: 230 patients in the control group and 220 patients in the intervention group. The comparison was made according to the risk factors of infection in the surgical sites. The incidence of infection was $7 \%$ in the case group and $15 \%$ in the control one $(p=0.011)$. They concluded from this study that using antimicrobial polyglactin-910 suture material can lead to saving health care resources as much as 1.5 million USD per year.

Recent studies in fields other than oral surgery have shown that TCS is an effective agent in reducing the bacterial growth on the skin and the incidence of postoperative infection due to its antimicrobial properties. However, there are contradictory studies in the field of oral surgery which have shown that antibacterial Vicryl suture not only is ineffective in reducing the number of gram-negative bacteria but also can have negative effects on oral normal flora; and thus, its' use is not recommended[13]. On the other hand, there are systematic review 
studies evaluating the effectiveness of Vicryl Plus suture against Vicryl suture material in which Meta-analysis showed a significant difference in surgical site infection; but based on low graded RCTs with a high risk of bias and imprecision[14].

Despite the extensive advertisement for Vicryl Plus suture materials; limited research has evaluated the effectiveness of this new product in surgeries involving mucosa. Besides, the different microbiological characteristics of the skin and mucosa and rinsing effect of saliva; can probably have an ablative effect on Vicryl Plus sutures leading to the nonsignificant clinical and microbiological difference between coated and non-coated sutures. Due to the all abovementioned factors, we decided to assess the efficacy of these suture materials in bacterial growth reduction in the oral cavity.

This study aimed to compare the efficiency of the Vicryl (non-coated) and Vicryl Plus (triclosan-coated) sutures in the reduction of the number and variety of bacterial species related to the manipulation of these suture materials in the oral cavity during the third molar surgery.

\section{Materials and Methods}

Calculation of the sample size (27 patients and 52 samples respectively) was done using the formula for Comparison of Two Means and a difference of $1.3 \times 10^{4}(\mathrm{CFU} / \mathrm{ml})$ was considered statistically significant with $\alpha$-error $=0.05$ and $1-\beta=0.80$. This study was performed as a double-blinded study in which none of the patient, surgeon, and microbiological technicians were aware of the exact location of Vicryl and antibacterial Vicryl sutures.

This study followed the Declaration of Helsinki on medical protocol and ethics and the Regional Ethical Review Board of Isfahan University of Medical Sciences approved the study on $7^{\text {th }}$ May 2019. All patients who participated in this study signed informed consent. 
The unwillingness of the patient to continue participation in the study came into consideration. All patients' information was kept confidential in this study. This study has been registered in the Iranian clinical trial registry (IRCT20131205015665N3).

Samples were selected among patients referred to the specialty section of the oral and maxillofacial department of the dentistry faculty of Isfahan University of Medical Sciences from May to July 2019. To be included in this study, patients should have had at least one mandibular third molar indicated for surgical extraction with the same degree of difficulty (type A mesioangular impaction) which was diagnosed through clinical and radiographic examination. Biographic data was taken (age, gender, address, and telephone number); and then, medical and dental history including pregnancy and breastfeeding, overall systemic condition, history of using medications in the last month, history of pulpitis or any malignant lesion in the site of surgery, were evaluated and recorded through clinical and radiographic examination. Patients with underlying systemic diseases, using systemic or oral medication affecting normal oral flora and colonization, smoking and drug addiction, alcohol addiction, presence of intraoral inflammation before the surgery, pregnancy, and lactation, known or suspected allergy to suture material or other materials used in this study, loss of sutures before 1 week, presence of removable prosthesis in the oral cavity, and presence of infection after surgery or any other condition indicated for antibiotic therapy were excluded from this study.

Considering an identification number (ID number) for each patient from 1 to 27 , similar sterile sets were used for all patients. The site of the surgery was prepared with betadine and draping was performed for all patients in the same manner. Local anesthesia was obtained for all patients with lidocaine $2 \%$ and epinephrine 1:80000. After reflecting a standard mucoperiosteal flap, bone removal and extraction of the tooth was performed and suturing was done with randomly placed Vicryl suture (Ethicon Inc., Johnson and Johnson Company, 
Somerville, New Jersey, United States) and Vicryl Plus suture (Ethicon Inc., Johnson, and Johnson Company, Somerville, New Jersey, United States) in the mesial and distal part of the surgical site. For randomization purposes, in patients with an odd ID number, Vicryl suture was placed in the mesial part and Vicryl Plus sutures in the distal part of the flap. In patients with an even ID number, the Vicryl Plus suture was placed in the mesial part of the flap, while the Vicryl suture was placed in the distal part. All the instruments including the injection needle, blade, elevator, and suture were sterile at the beginning. All procedures were performed by a single surgeon and, for blinding purposes; sutures were peeled open out of the surgeon's sight and then given to him. Cutting was done in a manner to permit $3 \mathrm{~mm}$ of the suture to be held out of the tissue. after considering a code for each type of suture material, the exact location of the suture at the surgical site was documented (i.e. "1M" means Vicryl suture in mesial and "2D" means Vicryl Plus suture in distal). Ibuprofen (soft gel capsule/400mg) was prescribed as a pain control medicament and extra gauze was given to the patient for application on the surgical site for 1 hour. Post-surgical patient instruction for oral hygiene was done. Patients were also instructed not to use any antiseptic mouth rinse for the first week after surgery before they were discharged. After seven days, recalling the patient and removing the sutures, placing each in a $5 \mathrm{cc}$ saline-filled test tube which had a code number on it as had been recorded at the date of the surgery. The tube tests were transported to the microbiological laboratory of Amin hospital in less than 1 hour. Patients were carefully evaluated for wound complications including bleeding and wound dehiscence and clinical examination was done assessing signs and symptoms of SSI post-surgically.

In the laboratory, after measuring the length of the removed sutures; they were transferred separately to the thioglycolate culture media and processed within 1 hour. For each sample, a one-tenth diluted solution with $0.9 \% \mathrm{NaCl}$ was made and $100 \mu \mathrm{L}$ of it was used on blood agar culture medium, and then Incubation was done in 37 degrees of temperature for 2 days. Gram 
coloring was done and cellular morphology was assessed under an optical microscope. $S$. mutans and Lactobacillus were considered as predominant bacterial species regarding the outcomes of similar studies evaluating the bacterial load following oral surgeries, which had reported these two species as predominant ones [15]. Counting the number of $S$. mutans and Lactobacillus colonies was done $(\mathrm{CFU} / \mathrm{mL})$ and evaluation of colonies with different morphology, color, size, and the hemolytic reaction was performed to determine the predominant bacterial species. For making measurements comparable, the mean number of colonies for S. mutans, Lactobacillus, and the total number of colonies were divided into the length of the suture material (CFU-length ratio) to get the number of colonies per length of suture for comparison. The decrease in the predominant species as well as total species was calculated by subtracting the number of species in the Vicryl Plus from the Vicryl sutures in each patient. The statistical method used in this study was descriptive analytics and Exact test performing with the SAS statistical software version 9.4 (SAS Institute, Cary, NC, USA).

\section{Results}

Three samples were lost in 3 patients when returned for suture removal appointment (one Vicryl Plus and two Vicryl sutures); the total number of samples was 51 from 27 patients with the mean age \pm SD of $23.7 \pm 3.1$ including six men $(22 \%)$ and 21 women $(78 \%)$. No wound complication including bleeding and wound dehiscence was detected after surgery and there was no clinical sign or symptom of SSI post-surgically. Two most frequent 1). The mean length of Vicryl bacterial species were S. mutans and Lactobacillus (Figure Plus $(5.97 \pm 2.37)$ suture was slightly greater than Vicryl (5.22 \pm 2.03$)$ suture but not statistically significant (paired t-test, $\mathrm{P}$-value=0.06). The mean $\mathrm{CFU}-$ length ratio $\pm \mathrm{SD}$ for the total bacterial species in the patients of our study for Vicryl and Vicryl Plus was $1.19 \pm 0.44$ and $1.03 \pm 0.31$ respectively. This ratio for $S$. mutans was $0.55 \pm 0.21$ and $0.52 \pm 0.17$ and for 
Figure 2). By using Vicryl Plus sutures, the total colonies of bacteria and Lactobacillus had decreased (compared to Vicryl) in 21 patients $(77.78 \%)$ and 20 patients $(74.07 \%)$ respectively ( $\mathrm{p}$ values $=0.005$ and 0.019 respectively) (Table 1 ). The decrease in the colonyforming unit-length (CFU-length) ratio in each patient can be seen in (Figure 3-5).

\section{Discussion}

Our results showed that using Vicryl Plus sutures which are coated with TCS in comparison with Vicryl in the oral surgery significantly reduced the total number of bacterial colonies' CFU length ratio as well as Lactobacillus colonies' CFU-length ratio; although it also decreased the S. mutans colonies' CFU-length ratio, this decrease was not statistically significant in the patients. Previous in vitro studies have shown that Vicryl Plus sutures can effectively inhibit the growth of staphylococcus aureus, methicillin-resistant staphylococcus aureus, staphylococcus epidermidis, and methicillin-resistant staphylococcus epidermidis, all are major microorganisms in surgical site infection in $\operatorname{skin}^{16}$. On the other hand, In a metaanalysis done by Guo et al. ${ }^{17}$ in 2016, the effect of TCS coated sutures on decreasing wound infection was evaluated. 13 randomized clinical trials with a total sample of 5256 patients were included. TCS group had a lower risk of surgical site infection (SSI) in every surgery in comparison with non-coated sutures (Risk Ratio $[\mathrm{RR}]=0.76$, Confidence Interval $[\mathrm{CI}]=0.65$ $0.88(95 \%), \mathrm{P}<0.001)$. The same ratio of wound opening was observed in two groups $(\mathrm{RR}=0.97, \mathrm{CI}=0.99-0.99 \quad(95 \%), \mathrm{P}=0.92)$. They concluded that TCS coated sutures can decrease the prevalence of SSI in abdominal surgeries. In another meta-analysis conducted by Edmiston et al.[8], 3568 patients in 13 RCTs were assessed showing the effectiveness of Vicryl Plus in reducing post-operative infections. Similar results were reported in Guo's and associates ${ }^{17}$ meta-analysis which was done in 2016 on 5268 patients; which do confirm outcomes of our work. 
Yet, there should be a more scientific base for judgment if TCS coated sutures has actual preventive effect on SSI incidence in the non-abdominal surgeries. The results of our study have shown that antibacterial Vicryl suture does have a reducing effect on bacterial load in the mandibular third molar extraction surgery as a non-abdominal surgery.

Storch et al. ${ }^{18}$ have reported a $96.7 \%$ decrease in S. aureus-related SSI after $48 \mathrm{~h}$ using Vicryl Plus; also, Ming et al. ${ }^{19}$ in a similar study, reported a decrease in $S$. aureus and E. coli species using Monocryl plus. Alonso et al. ${ }^{20}$ expressed an almost $87 \%$ decrease in infection by $S$. epidermidis and E. coli in 16 patients receiving Vicryl Plus sutures. Finally, Marco et al. ${ }^{21}$ reported a $66 \%$ decrease in S. epidermidis-related SSI in rats. World Health Organization (WHO) has also recommended using triclosan-coated sutures ${ }^{22}$ and they are proven to be financially affordable ${ }^{16}$. Despite the histological and microbiological differences in surgical sites (skin vs. Mucosa), exposure to food particles and constant secretion of saliva, and also being an environment influenced by breathing process all which can have an ablative effect on the efficiency of the antibacterial suture materials; our study has approved the abovementioned results and showed that Vicryl Plus can decrease the bacterial load in the oral surgical site. It is reported in several other studies that Vicryl Plus can be an effective tool reducing the rate of post-operative infection in abdominal ${ }^{17,23}$ or medullary ${ }^{24}$ surgeries; which are in accordance with the results of our study.

Although many studies are in favor of our results, there is some degree of contradiction in some other studies ${ }^{25-27}$. In one study performed by Tabrizi et al. ${ }^{28}$, comparing the ability of TCS coated sutures to reduce the infection in 320 patients undergone implant surgery, no difference were reported between Vicryl and Vicryl Plus sutures. One probable reason may be the fact that one important requisite for patients undergoing implant surgery, is to have excellent oral hygiene. The site of the surgery may be another factor that can be considered; since the site of surgery in mandibular wisdom tooth extraction located in the most posterior 
aspect of the mouth, less accessibility before and after surgery, this fact can make it more prone to SSI. Thus, antibacterial suture materials including Vicryl Plus can be a useful tool for the reduction of bacterial load and subsequent surgical site infection in cases with a high risk of infection due to the patient-related factors (i.e. poor oral hygiene).

In another study with split-mouth design, after surgical extraction of both impacted mandibular wisdom teeth simultaneously, suturing was performed using Monocryl plus or silk in each side and microbiological evaluation was done 72 hours and 7 days after surgery. The most common bacterial species were gram-positive cocci, gram-positive bacillus (lactobacillus), and gram-negative bacillus (prevotella). Only one case of SSI was reported after $72 \mathrm{~h}$. Utmost antibacterial effect of Monocryl plus suture was after $72 \mathrm{~h}$, but the difference was not significant after 5-7 days which is the routine time of suture removal in everyday practice in dentistry ${ }^{15}$, and also our study, but the results are in contrast which may be due to the difference in suture materials between two studies; the reason may be the loss of antibacterial effect of Monocryl plus after $72 \mathrm{~h}$ while Vicryl Plus may have a longer antibacterial effect in the similar situation. It seems that performing a similar study comparing these two suture materials can help assess this theory.

In an in vitro study by Venema et al. ${ }^{25}$, Vicryl Plus had no inhibitory effect on Streptococcus sanguis PK1889 or other microorganisms residing in saliva. This study is in contrast with our results. This might be due to the differences in the method of performing these two studies and in vitro nature of Venema's et al. study versus in vivo nature of our study.

Our study had certain strengths and limitations. The strength of our study is the random allocation as well as having a parallel design with two simultaneous interventions in each participant which makes the results more reliable by having control over confounding variables when compared to a design with separate case and control groups. As per the 
limitation of our study, since the number of female participants was noticeably greater than male participants (21 versus 6$)$ the results of this study cannot be generalized in terms of gender. Moreover, further studies with more samples can confirm the results of this study and clarify the true usefulness of Vicryl Plus sutures in the reduction of bacterial colonies in the surgical field. Increasing the period of follow up from two to four weeks could have also increased the validity of our study and is suggested for the future studies. The longer period of follow up other antibacterial sutures and the comparison between them can also help the dentists to choose the best suture material in oral surgeries. The selection of aerobic species as well as other common anaerobic species other than those evaluated in our study can increase the accuracy of future studies. Besides, performing other culturing and coloring techniques which were not included in our study due to their complexity and high price can be a good suggestion.

\section{Conclusion:}

The results of our study have shown that Vicryl Plus suture is more beneficial than Vicryl suture in bacterial load reduction after mandibular third molar extraction surgery. This might benefit especially high-risk patients with poor oral hygiene.

Acknowledgments: The results have been extracted from a thesis submitted to the school of Dentistry, Isfahan University of Medical Sciences, in partial fulfillment of the requirement for the DDS degree of Mrs. Sameen Rahgozar.

\section{Funding Information:}

This study was funded by The Research Council of Isfahan University of Medical Sciences (Grant \#397711).

\section{References:}


1. Kautto, A.; Vehkalahti, M.M.; Ventä, I.Age of patient at the extraction of the third molar. Int. J. Oral Maxillofac. Surg. 2018, 47, 947-951, doi:10.1016/j.ijom.2018.03.020.

2. Velnar, T.; Bailey, T.; Smrkolj, V.The wound healing process: An overview of the cellular and molecular mechanisms. J. Int. Med. Res. 2009, 37, 1528-1542.

3. Singh, S.; Young, A.; McNaught, C.-E.The physiology of wound healing. Surg. 2017, 35, doi:10.1016/j.mpsur.2017.06.004.

4. Javed, F.; Al-Askar, M.; Almas, K.; Romanos, G.E.; Al-Hezaimi, K.Tissue reactions to various suture materials used in oral surgical interventions. ISRN Dent. 2012, 2012, 762095, doi:10.5402/2012/762095.

5. Grigg, T.R.; Liewehr, F.R.; Patton, W.R.; Buxton, T.B.; McPherson, J.C.Effect of the Wicking Behavior of Multifilament Sutures. J. Endod. 2004, 30, 649-652, doi:https://doi.org/10.1097/01.DON.0000121617.67923.05.

6. Banche, G.; Roana, J.; Mandras, N.; Amasio, M.; Gallesio, C.; Allizond, V.; Angeretti, A.; Tullio, V.; Cuffini, A.Microbial Adherence on Various Intraoral Suture Materials in Patients Undergoing Dental Surgery. J. Oral Maxillofac. Surg. 2007, 65, 15031507, doi:10.1016/j.joms.2006.10.066.

7. Justinger, C.; Moussavian, M.R.; Schlueter, C.; Kopp, B.; Kollmar, O.; Schilling, M.K.Antibiotic coating of abdominal closure sutures and wound infection. Surgery 2009, 145, 330-334, doi:https://doi.org/10.1016/j.surg.2008.11.007.

8. Edmiston, Charles, J.; Seabrook, G.; Goheen, M.; Krepel, C.; Johnson, C.; Lewis, B.; Brown, K.; Towne, J.Bacterial Adherence to Surgical Sutures: Can AntibacterialCoated Sutures Reduce the Risk of Microbial Contamination? J. Am. Coll. Surg. 2006, 203, 481-489, doi:10.1016/j.jamcollsurg.2006.06.026.

9. Dragović, M.; Pejovic, M.; Stepic, J.; Dragovic, S.; Nikolic, N.; Kuzmanovic Pficer, J.; Colic, S.; Milasin, J.Microbial adherence affinity and clinical characteristics of polypropylene versus silk sutures in oral surgery. Srp. Arh. Celok. Lek. 2018, 146, 258-263, doi:10.2298/SARH170428184D.

10. Cruz, F.; Leite, F.; Cruz, G.; Cruz, S.; Reis, J.; Pierce, M.; Cruz, M.Sutures coated with antiseptic pomade to prevent bacterial colonization: a randomized clinical trial. 
Oral Surg. Oral Med. Oral Pathol. Oral Radiol. 2013, 116, e103-9, doi:10.1016/j.oooo.2011.12.016.

11. Russell, A.D.Whither triclosan? J. Antimicrob. Chemother. 2004, 53, 693-695, doi:10.1093/jac/dkh171.

12. Galal, I.; El-Hindawy, K.Impact of using triclosan-antibacterial sutures on incidence of surgical site infection. Am. J. Surg. 2011, 202, 133-138, doi:10.1016/j.amjsurg.2010.06.011.

13. Pelz, K.; Tödtmann, N.; Otten, J.E.Comparison of antibacterial-coated and non-coated suture material in intraoral surgery by isolation of adherent bacteria. Ann. Agric.

Environ. Med. 2015, 22, 551-555, doi:10.5604/12321966.1167733.

14. Wu, X.; Kubilay, N.Z.; Ren, J.; Allegranzi, B.; Bischoff, P.; Zayed, B.; Pittet, D.; Li, J.Antimicrobial-coated sutures to decrease surgical site infections: a systematic review and meta-analysis. Eur. J. Clin. Microbiol. Infect. Dis. 2017, 36, 19-32, doi:10.1007/s10096-016-2765-y.

15. Sala-Pérez, S.; López-Ramírez, M.; Quinteros-Borgarello, M.; Valmaseda-Castellón, E.; Gay-Escoda, C.Antibacterial suture vs silk for the surgical removal of impacted lower third molars. A randomized clinical study. Med. Oral Patol. Oral Cir. Bucal 2016, 21, e95-e102, doi:10.4317/medoral.20721.

16. Rothenburger, S.; Spangler, D.; Bhende, S.; Burkley, D.In Vitro Antimicrobial Evaluation of Coated VICRYL* Plus Antibacterial Suture (Coated Polyglactin 910 with Triclosan) using Zone of Inhibition Assays. Surg. Infect. (Larchmt). 2002, 3 Suppl 1, S79-87, doi:10.1089/10962960260496361.

17. Guo, J.; Pan, L.-H.; Li, Y.-X.; Yang, X.-D.; Li, L.-Q.; Zhang, C.-Y.; Zhong, J.H.Efficacy of triclosan-coated sutures for reducing risk of surgical site infection in adults: a meta-analysis of randomized clinical trials. J. Surg. Res. 2016, 201, 105-117, doi:https://doi.org/10.1016/j.jss.2015.10.015.

18. Storch, M.; Perry, L.; Davidson, J.; Ward, J.A 28-Day Study of the Effect of Coated VICRYL* Plus Antibacterial Suture (Coated Polyglactin 910 Suture with Triclosan) on Wound Healing in Guinea Pig Linear Incisional Skin Wounds. Surg. Infect.

(Larchmt). 2002, 3 Suppl 1, S89-98, doi:10.1089/10962960260496370. 
19. Ming, X.; Nichols, M.; Rothenburger, S.In vivo antibacterial efficacy of MONOCRYL plus antibacterial suture (Poliglecaprone 25 with triclosan). Surg. Infect. (Larchmt).

2007, 8, 209-214, doi:10.1089/sur.2006.004.

20. Alonso, A.; García-Criado, F.; Parreno, F.; Garcia Sanchez, E.; Sanchez, J.E.; ParreñoManchado, A.; Zambrano, Y.Study of the efficacy of Coated VICRYL Plus ${ }^{\circledR}$ Antibacterial suture (coated Polyglactin 910 suture with Triclosan) in two animal models of general surgery. J. Infect. 2007, 54, 82-88, doi:10.1016/j.jinf.2006.01.008.

21. Marco, F.; Vallez, R.; Gonzalez, P.; Ortega, L.; Lama, J.; Stern, L.Study of the Efficacy of Coated Vicryl Plus ${ }^{\circledR}$ Antibacterial Suture in an Animal Model of Orthopedic Surgery. Surg. Infect. (Larchmt). 2007, 8, 359-365, doi:10.1089/sur.2006.013.

22. Allegranzi, B.; Zayed, B.; Bischoff, P.; Kubilay, N.Z.; deJonge, S.; deVries, F.; Gomes, S.M.; Gans, S.; Wallert, E.D.; Wu, X.; et al.New WHO recommendations on intraoperative and postoperative measures for surgical site infection prevention: an evidence-based global perspective. Lancet Infect. Dis. 2016, 16, e288-e303, doi:https://doi.org/10.1016/S1473-3099(16)30402-9.

23. Henriksen, N.; Deerenberg, E.; Venclauskas, L.; Fortelny, R.; Garcia-Alamino, J.M.; Miserez, M.; Muysoms, F.Triclosan-coated sutures and surgical site infection in abdominal surgery: the TRISTAN review, meta-analysis and trial sequential analysis. Hernia 2017, 21, doi:10.1007/s10029-017-1681-0.

24. Ueno, M.; Saito, W.; Yamagata, M.; Imura, T.; Inoue, G.; Nakazawa, T.; Takahira, N.; Uchida, K.; Fukahori, N.; Shimomura, K.; et al.Triclosan-coated sutures reduce wound infections after spinal surgery: A retrospective, nonrandomized, clinical study. Spine J. 2013, 15, doi:10.1016/j.spinee.2013.06.046.

25. Venema, S.; Abbas, F.; van deBelt-Gritter, B.; van derMei, H.C.; Busscher, H.J.; vanHoogmoed, C.G.In Vitro Oral Biofilm Formation on Triclosan-Coated Sutures in the Absence and Presence of Additional Antiplaque Treatment. J. Oral Maxillofac. Surg. 2011, 69, 980-985, doi:https://doi.org/10.1016/j.joms.2010.02.030.

26. Mingmalairak, C.; Ungbhakorn, P.; Paocharoen, V.Efficacy of antimicrobial coating suture coated polyglactin 910 with tricosan (Vicryl Plus) compared with polyglactin 
910 (Vicryl) in reduced surgical site infection of appendicitis, double blind randomized control trial, preliminary safety report. J. Med. Assoc. Thai. 2009, 92, 770-775.

27. Ford, H.; Jones, P.; Gaines, B.; Reblock, K.; Simpkins, D.Intraoperative Handling and Wound Healing: Controlled Clinical Trial Comparing Coated VICRYL® Plus Antibacterial Suture (Coated Polyglactin 910 Suture with Triclosan) with Coated VICRYL® Suture (Coated Polyglactin 910 Suture). Surg. Infect. (Larchmt). 2005, 6, 313-321, doi:10.1089/sur.2005.6.313.

28. Tabrizi, R.; Mohajerani, H.; Bozorgmehr, F.Polyglactin 910 suture compared with polyglactin 910 coated with triclosan in dental implant surgery: randomized clinical trial. Int. J. Oral Maxillofac. Surg. 2019, 48, 1367-1371, doi:https://doi.org/10.1016/j.ijom.2019.01.011. 
Table 1- Changes in the CFU/length in different bacterial colonies by using Vicryl Plus (Vicryl Plus minus Vicryl)

\begin{tabular}{|c|c|c|c|c|c|}
\hline & & \multirow[t]{2}{*}{$\mathbf{N}(\%)$} & \multirow{2}{*}{$\begin{array}{c}\mathrm{P}^{*} \\
\text { value }\end{array}$} & \multicolumn{2}{|l|}{ age } \\
\hline & & & & Mean \pm SD & P-value \\
\hline \multirow{2}{*}{$\begin{array}{l}\text { Total species of } \\
\text { bacteria }\end{array}$} & Same or increase & $6(22.22)$ & \multirow[t]{2}{*}{0.005} & $23.50 \pm 2.94$ & \multirow[t]{2}{*}{0.837} \\
\hline & Decrease & $21(77.78)$ & & $23.80 \pm 3.29$ & \\
\hline \multirow[t]{2}{*}{ S. mutans } & Same or increase & $12(44.44)$ & \multirow[t]{2}{*}{0.701} & $24 \pm 3.24$ & \multirow[t]{2}{*}{0.711} \\
\hline & Decrease & $15(55.56)$ & & $23.53 \pm 3.20$ & \\
\hline \multirow[t]{2}{*}{ Lactobacillus } & Same or increase & $7(25.93)$ & \multirow[t]{2}{*}{0.019} & $24.28 \pm 3.40$ & \multirow[t]{2}{*}{0.606} \\
\hline & Decrease & $20(74.07)$ & & $23.55 \pm 3.15$ & \\
\hline
\end{tabular}

Figure 1Percentage of the bacterial species following gram coloring in Vicryl and Vicryl Plus groups

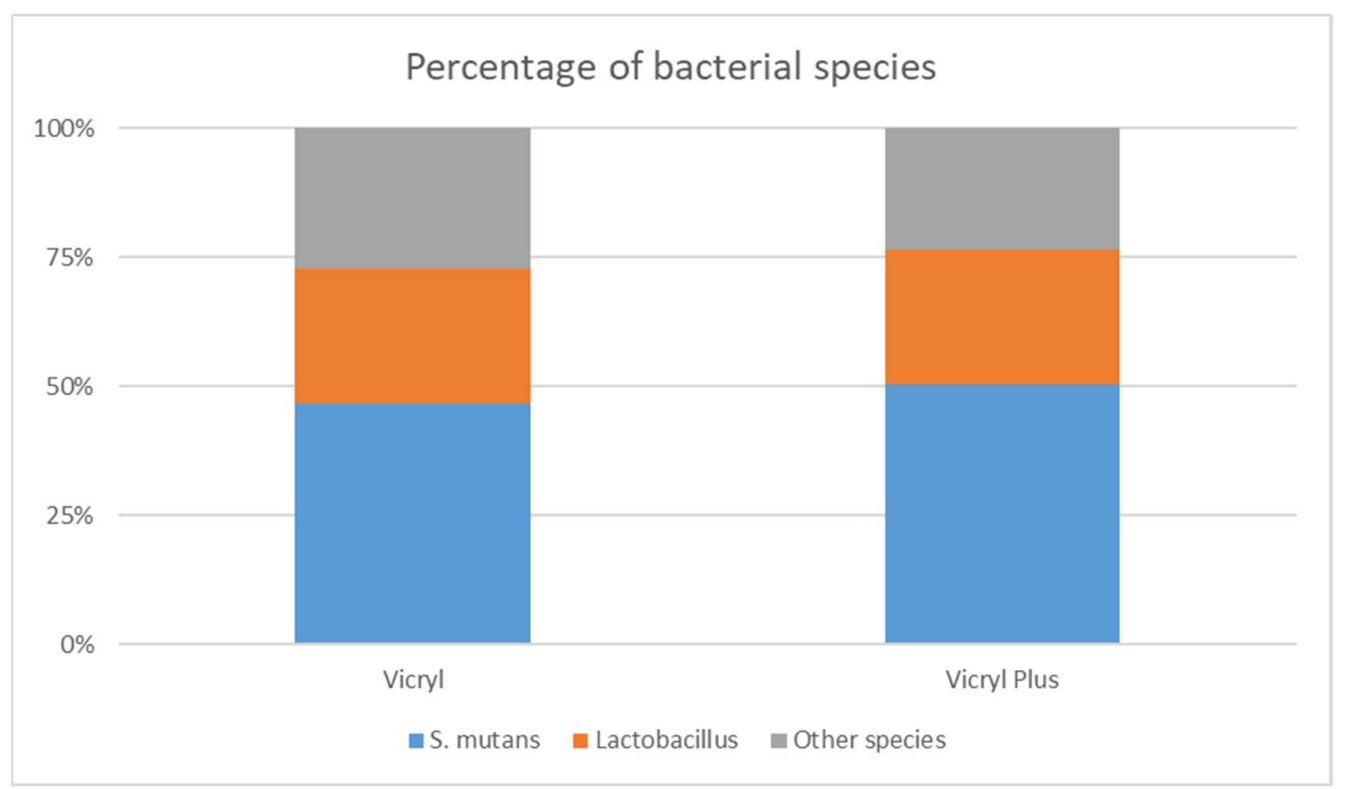

Figure 2-Comparison of the CFU-length ratio of different bacterial species in Vicryl and Vicryl Plus sutures 




Figure 3 Decrease in the CFU-length ratio regarding the total bacterial species (calculated by subtracting CFU-length ratio of Vicryl Plus from Vicryl in each patient)



Figure 4 Decrease in the CFU-length ratio regarding S. Mutans (calculated by subtracting CFU-length ratio of Vicryl Plus from Vicryl in each patient)

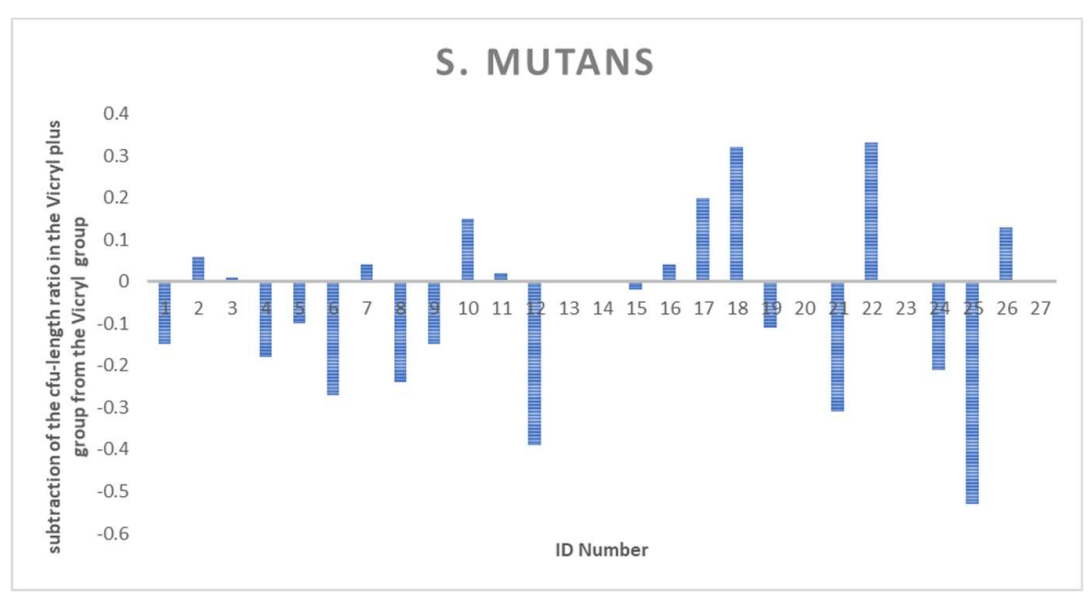


Figure 5 Decrease in the CFU-length ratio regarding Lactobacillus. (Calculated by subtracting CFU-length ratio of Vicryl Plus from Vicryl in each patient)

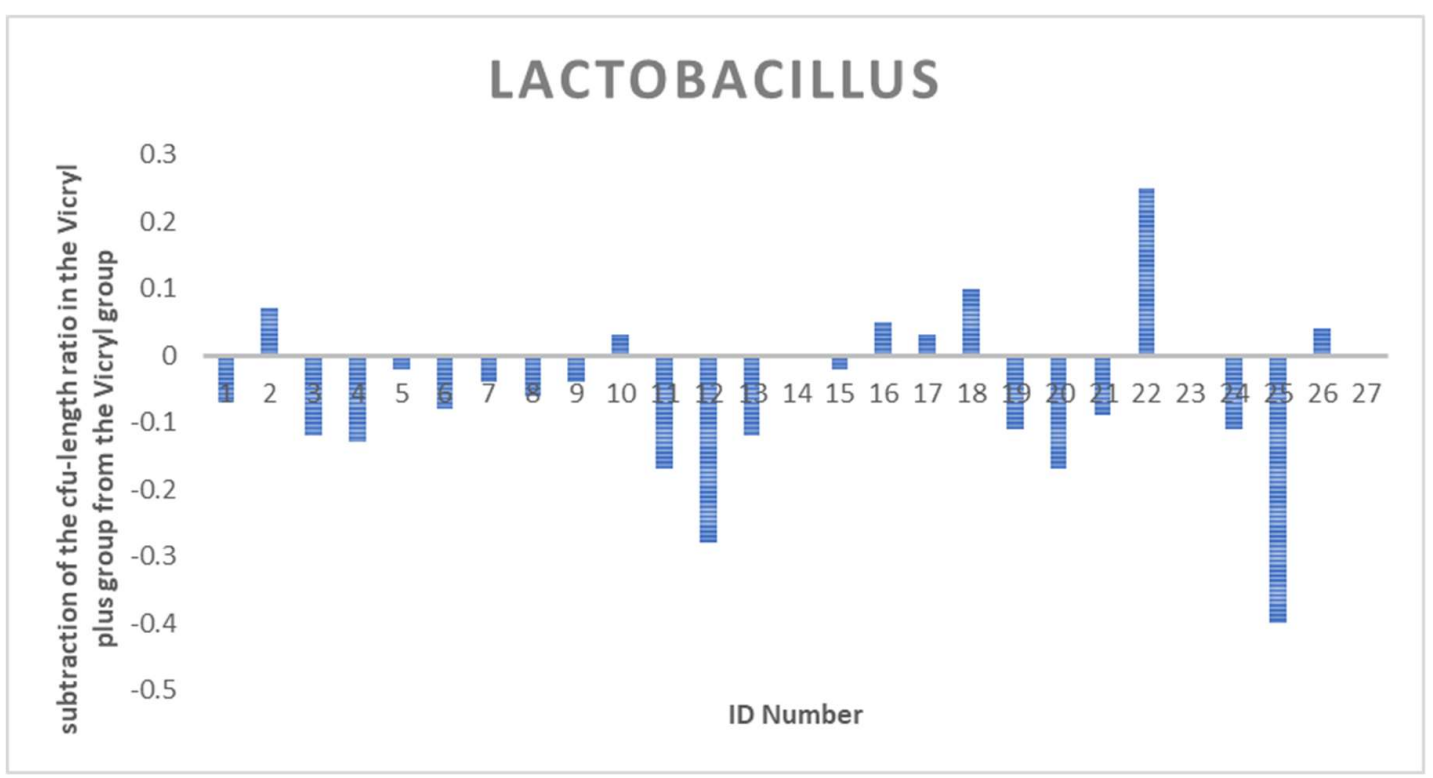

